\title{
Lipogenesis in a wing-polymorphic cricket: canalization versus morph-specific plasticity as a function of nutritional heterogeneity
}

Anthony J. Zera ${ }^{1,3}$, Rebecca Clark ${ }^{1}$, and Spence Behmer ${ }^{2}$ 


\section{Abstract}

20 The influence of variable nutritional input on life history adaptation is a central, but

21 incompletely understood aspect of life history physiology. The wing-polymorphic cricket,

22 Gryllus firmus, has been extensively studied with respect to the biochemical basis of life

23 history adaptation, in particular, modification of lipid metabolism that underlies the enhanced

24 accumulation of lipid flight fuel in the dispersing morph $[(\operatorname{LW}(f)=$ long wings with functional

25 flight muscles] relative to the flightless ( $\mathrm{SW}=$ short-winged) morph. To date, biochemical

26 studies have been undertaken almost exclusively using a single laboratory diet. Thus, the

27 extent to which nutritional heterogeneity, likely experienced in the field, influences this key

28 morph adaptation is unknown. We used the experimental approach of the Geometric

29 Framework for Nutrition and employed 13 diets that differed in the amounts and ratios of

30 protein and carbohydrate to assess how nutrient amount and balance affects morph-specific

31 lipid biosynthesis. Greater lipid biosynthesis and allocation to the soma in the LW(f)

32 compared with the SW morph (1) occurred across the entire protein-carbohydrate

33 landscape and (2) is likely an important contributor to elevated somatic lipid in the LW(f)

34 morph across the entire protein-carbohydrate landscape. Nevertheless, dietary

35 carbohydrate strongly affected lipid biosynthesis in a morph-specific manner (to a greater

36 degree in the $L W(f)$ morph). Lipogenesis in the SW morph may be constrained due to its

37 more limited lipid storage capacity compared to the LW(f) morph. Elevated activity of

$38 \mathrm{NADP}^{+}$-isocitrate dehydrogenase $\left(\mathrm{NADP}^{+}-\mathrm{IDH}\right)$, an enzyme that produces reducing

39 equivalents for lipid biosynthesis, was correlated with and may be an important cause of the

40 increased lipogenesis in the LW(f) morph across most, but not all regions of the protein-

41 carbohydrate landscape. By contrast, ATP-citrate lyase (ACL), an enzyme that catalyzes

42 the first step in the pathway of fatty acid biosynthesis, showed complex morph-specific

43 patterns of activity that were strongly contingent upon diet. Morph-specific patterns of 
$44 \mathrm{NADP}^{+}-\mathrm{IDH}$ and $\mathrm{ACL}$ activities across the nutrient landscape were much more complex

45 than expected from previous studies on a single diet. Collectively, our results indicate that

46 the biochemical basis of an important life history adaptation, morph-specific lipogenesis,

47 can be canalized in the face of substantial nutritional heterogeneity. However, in some

48 regions of the protein-carbohydrate landscape, it is strongly modulated in a morph-specific

49 manner.

50

51

52

53

54

55

56 


\section{Introduction}

58 Wing polymorphism is a common feature of insects, consisting of flight-capable and flightless

59 morphs within the same population that are adapted for dispersal at the expense of reproductive

60 output, and vice versa (Harrison, 1980; Zera and Denno, 1997; Guerra, 2011; Zera and Brisson,

61 2012). The polymorphism has been extensively studied from ecological, behavioral,

62 physiological, biochemical, and molecular perspectives to understand the functional basis of

63 morph adaptation for dispersal versus reproduction. It has also served as a major experimental

64 model in functional studies of life history trade-offs, a central topic in life history evolution (see

65 above references).

66 Physiological and biochemical aspects of the polymorphism have been especially well-

67 studied in the wing-dimorphic cricket, Gryllus firmus (reviewed in Zera, 2005, 2009; Zera and

68 Harshman, 2011; Schilder, et al., 2011; Vellichirammal et al., 2014). This species consists of a

69 flight-capable morph with fully developed wings and flight muscles [LW(f) = long wings and

70 functional flight muscles] and an obligately flightless morph that does not fully develop wings or

71 flight muscles and which cannot fly (SW = short-winged). A key difference between the morphs

72 is the significantly greater accumulation of lipid (main flight fuel) by the LW(f) morph during

73 adulthood. This is due to elevated expression of genes and activities of lipogenic enzymes that

74 result in elevated flux through the pathways of fatty-acid and triglyceride biosynthesis in LW(f)

75 adults (Zhao and Zera, 2002; Zera and Zhao, 2003; Zera 2005; Zera and Harshman, 2011;

76 Vellichirammel et al., 2014). Importantly, reduced lipid production in the SW morph allows this

77 morph to allocate a greater proportion of ingested nutrients (e.g., protein and carbohydrates) to

78 egg production, accounting, at least in part, for its substantially elevated fecundity, relative to the

79 LW(f) morph (see above references).

80 Despite considerable study, many important aspects of morph-specific lipid metabolism

81 have yet to be investigated in G. firmus morphs. One potentially-important, but neglected,

82 aspect is how variable food nutritional input influences morph-specific lipid biosynthesis and its 
83 trade-off with egg production. The role of variable nutrient acquisition on life history trade-offs is 84 a long-standing issue that has become an increasingly important focus of life history physiology 85 (van Noordwijk and de Jong, 1986; Zera and Harshman, 2001; Lee et al., 2008; Boggs, 2009; 86 Flatt and Heyland, 2011; Musselman et al., 2013). To date, nearly all previous physiological, 87 biochemical and molecular studies of lipid metabolism in G. firmus have been conducted on a 88 single diet, or in a limited number of studies, the total nutrient content of a single diet was 89 altered (e.g. reduced by 50-75\%; e.g. Zhao and Zera, 2002; Zera and Zhao, 2003, 2006; Zera 90 and Harshman, 2011). Thus, we currently have a very narrow understanding of the effects of 91 food nutrient content on the biochemical basis of life history specialization and trade-offs.

92 One effective way to investigate the influence of food nutritional heterogeneity on various 93 organismal functions is to employ the experimental approach of the Geometric Framework for 94 Nutrition (Simpson and Raubenheimer, 1999, 2012; Behmer, 2009). In essence, this approach 95 examines animal responses over a range of foods that differ in specific nutrients. Typically 96 protein and carbohydrate content are the nutrients that are manipulated because they have very

97 strong effects on growth and reproduction, and are actively regulated by most animals, including 98 insects (Behmer, 2009). Response variables (e.g., consumption, respiration, lipid accumulation, 99 various life history traits) are then measured as a function of food protein-carbohydrate content.

100 Because a relatively large number of diets are often used in these studies (e.g., 13 in the 101 present study; see below), quantitative effects of variation in food protein and carbohydrate 102 amounts and balance, as well as total caloric content, can be assessed. This experimental 103 approach thus overcomes many of the limitations of previous studies of life history nutritional 104 physiology, in which the nutritional environment (i.e., variation in diet) has not been well 105 quantified or controlled, and where the nutrient space was severely restricted because only a 106 few diets were used (Lee et al., 2008; and references therein).

107 We recently demonstrated, using the experimental approach of the Geometric 108 Framework, that LW(f) adult G. firmus females have significantly greater somatic lipid content 
109 than SW females over a wide protein-carbohydrate landscape, involving diets that differ in

110 carbohydrate and protein (see Discussion, and Clark et al., 2015). This result extended our

111 previous findings of greater somatic lipid in LW(f) vs. SW females fed a single diet. However,

112 the underlying biochemical causes for this finding were unknown. In the present study we

113 reared flight-capable adult female morphs of Gryllus firmus on a wide range of diets that differed

114 in their protein-carbohydrate content, and then quantified key aspects of lipid biosynthesis and

115 allocation to somatic and reproductive body compartments. Specifically, we measured the rate

116 of whole-organism lipid biosynthesis - using a radiolabeled lipid precursor $\left({ }^{14} \mathrm{C}\right.$-acetate $)$ - and

117 allocation of biosynthesized lipid to somatic and ovarian body compartments in LW(f) and SW

118 adult females. In addition, we quantified specific activities of two important lipogenic enzymes:

119 (1) $\mathrm{NADP}^{+}$-isocitrate dehydrogenase $\left(\mathrm{NADP}^{+}-\mathrm{IDH}\right)$, which is an important contributor of reduced

120 NADPH required for lipogenesis and (2) ATP-citrate lyase (ACL), which catalyzes the first step

121 in the de novo pathway of fatty acid biosynthesis (Zera, 2005). Activities of these two enzymes

122 are strongly and positively correlated genetically with each other, with activities of three other

123 lipogenic enzymes, and with rate of lipid biosynthesis in wing morphs of G. firmus fed the

124 standard laboratory diet (Zera and Zhao, 2003; Zera, 2005). The present study used 13

125 precisely defined diets that varied in the total amount and balance of protein and carbohydrate

126 content. This array of diets allowed us to investigate how the magnitude of morph-specific

127 variation in lipid biosynthesis and ovarian growth, and their trade-off, varied across a broad

128 protein-carbohydrate landscape.

129

130 Methods

131 Crickets and diets. Gryllus firmus females used in the present study were derived from two

132 large outbred laboratory populations: one population primarily (>90\%) produces the flight-

133 capable morph with long wings and fully developed flight muscles [LW(f)], while the other

134 primarily (>95\%) produces the SW flightless morph with shortened nonfunctional wings and 
135 underdeveloped non-functional flight muscles. Some LW(f) individuals are transformed into a 136 flightless morph during adulthood due to histolysis of flight muscles [denoted as the $\operatorname{LW}(\mathrm{h})$

137 morph; Zera, 2009]. This morph, which has reproductive, physiological, and biochemical 138 characteristics more similar to SW females than LW(f) females (Zera, 2009), is not considered 139 in the present study. Our Gryllus firmus populations were founded from individuals collected at 140 Gainesville, Florida in the mid-1990s and have been maintained since then in the laboratory 141 under a 16 light: 8 dark photo regime, at $28^{\circ} \mathrm{C}$ and fed a standard laboratory diet (Zera, 2005).

142 Each population was propagated each generation by breeding 100-250 individuals. These 143 populations, which are part of a larger group of populations produced by artificial selection, are 144 the same as those investigated previously in numerous biochemical and physiological studies of 145 morph adaptation (e.g. Zera and Larsen, 2001; Zhao and Zera, 2002; Zera and Zhao 2003, 146 2006; reviewed in Zera and Harshman, 2009, 2011). Although only one pair of LW(f) and SW 147 lines were investigated in the present study, previous studies have shown that biochemical and 148 reproductive differences between any pair of $L W(f)$ and SW lines are typically very similar to 149 differences between of other $\mathrm{LW}(\mathrm{f})$ and SW lines (see above references). Biochemical data 150 analogous to those reported here will be reported for the other LW(f) and SW selected lines in 151 forthcoming papers. Additional information on these populations can be found in references 152 cited above.

153 Individuals that had molted into adults during the previous 24 hours were transferred 154 from the standard laboratory diet to plastic boxes (14" x 9" x 6'; 6-12 crickets per box) that 155 contained one of 13 chemically-defined diets that differed in protein (p) or carbohydrate (c) 156 content (see Appendix 1, and Clark et al. (2015) for information on diet composition). Previous 157 work has shown that these crickets self-select a protein-to-carbohydrate ratio of p3:c4; this 158 "balanced" ratio was used along with two ratios that were protein-biased ( $117.25: c 14.25$ and $159 \mathrm{p} 14: \mathrm{c} 7)$ and two ratios that were carbohydrate-biased (p4:c17 and p9.75:c21.75; Clark et al., 160 2013). In addition, for each p:c ratio, two or three total macronutrient contents were used, so 
161 that total diet macronutrient content ranged from dilute (21\% total macronutrients) to

162 concentrated (63\%). Food was placed in plastic dishes and was continually replenished during

163 the experiment (see Clark et al., 2013). Boxes also contained two $50 \mathrm{~mL}$ cotton-plugged plastic

164 vials containing water. Both $\mathrm{LW}(\mathrm{f})$ and SW females were raised in the same box. Boxes were

165 kept at $28^{\circ} \mathrm{C}, 16 \mathrm{~L}: 8 \mathrm{D}$ for five days at which point crickets were weighed and injected with

166 radiolabeled $\left[1-{ }^{14} \mathrm{C}\right] \mathrm{Na}$-acetate as described below. In a few instances, a cricket was

167 cannibalized during the 5-day period prior to injection. Where this occurred the remaining

168 crickets in that box were eliminated from the study.

170 Quantification of whole body lipid biosynthesis. Incorporation of radiolabelled $\left[1-{ }^{14} \mathrm{C}\right] \mathrm{Na}-$ 171 acetate into lipid was performed essentially as in Zhao and Zera (2001, 2002), with a few slight 172 modifications. Briefly, about 800,000-900,000 DPM of $\left[1-{ }^{14} \mathrm{C}\right] \mathrm{Na}$-acetate $(2.0 \mathrm{Gbq} / \mathrm{mmol} ; 54.7$ $173 \mathrm{mCi} / \mathrm{mmol}$ ) in $3 \mu \mathrm{l}$ of $0.9 \% \mathrm{NaCl}$ solution were injected in the abdominal hemocoel of a cricket.

174 Injections were performed within 2-4 hours after lights on, in crickets that had been starved for 2 $175 \mathrm{~h}$ prior to injection. Any hemolymph that bled at the site of injection was wiped with a small piece 176 of adsorbent paper; radioactivity on this paper was quantified by liquid scintillation spectrometry, 177 and subtracted from DPM injected. Each day, DPM in three replicates of the injection solution 178 were also determined so that percent incorporation of radiolabel into lipid could be calculated. 179 After injection, crickets were placed singly in 16 oz (ca $500 \mathrm{~mL}$ ) plastic cups and kept at $28^{\circ} \mathrm{C}$ $18016 \mathrm{~L}: 8 \mathrm{D}$ for $3 \mathrm{~h}$ (this was within the linear range of incorporation of radiolabel into total lipid 181 versus time (see Fig 1 of Zhao and Zera, 2001)). After the incubation period, crickets were 182 quickly frozen at $-86^{\circ} \mathrm{C}$.

183 Crickets were subsequently thawed and weighed, flight muscle status scored (pink or 184 white), and ovaries removed and weighed. Flight muscle status was recorded because, as 185 mentioned previously, some LW(f) individuals histolyze their flight muscles and become 186 flightless; results for $\mathrm{LW}(\mathrm{h})$ females will be reported elsewhere. To determine how much 
187 radiolabel was incorporated into somatic and reproductive tissues, ovaries were removed and

188 placed in $1.5 \mathrm{~mL}$ Eppendorf tubes, to which were added $500 \mu \mathrm{L}$ of chloroform/methanol $(2: 1$

$189 \mathrm{v} / \mathrm{v})$. Cricket bodies without ovaries were placed individually in glass tubes, to which were added

$1905 \mathrm{~mL}$ of chloroform/methanol $(2: 1, \mathrm{v} / \mathrm{v})$. Tubes containing ovaries or cricket bodies were stored

191 at $-20^{\circ} \mathrm{C}$ until lipid extraction. Bodies were homogenized for $2 \mathrm{~min}$ with a glass rod attached to

192 an electric motor, and the mixture was filtered through Whatman \#1 paper. The residue was

193 returned to the tube and the process was repeated. The total chloroform/methanol extract was

194 washed with $1 / 4$ of its volume with $0.88 \%$ aqueous $\mathrm{KCl}$, followed by two additional washes of

$1950.88 \% \mathrm{KCl}$ in water $\left(1 / 8^{\text {th }}\right.$ of extract volume $)$ and methanol $\left(1: 1, \mathrm{v} / \mathrm{v} ; 1 / 8^{\text {th }}\right.$ of extract volume $)$

196 These washes were done to remove unreacted radiolabeled Na-acetate as well as

197 contaminating chloroform-soluble carbohydrates. The volume of the washed

198 chloroform/methanol solution was reduced to $3 \mathrm{~mL}, 2.7 \mathrm{~mL}$ of which were placed in scintillation

199 vials. The solutions were evaporated to dryness on a heating block, three $\mathrm{mL}$ of scintillation

200 cocktail were added, the tubes were kept in the dark overnight to reduce chemiluminescence,

201 and DPM quantified the next day. Ovarian lipids were extracted in a similar way as somatic lipid,

202 except that ovaries in chloroform/methanol were sonicated for ten seconds, centrifuged at

$20313,000 \times g$, and organic solvent removed. This process was repeated, the extracts were

204 combined and washed with aqueous $\mathrm{KCl}$, and the lower organic phase was removed and

205 counted as described above. DPM incorporated into total lipid were divided by total DPM

206 injected to obtain \% injected radiolabel incorporated into lipid.

208 Quantification of enzyme activities. Fat body specific activities of $\mathrm{NADP}^{+}$-isocitrate

209 dehydrogenase (NADP ${ }^{+}$-IDH; E.C. 1.1.1.42) and ATP-citrate lyase (E.C. 4.1.3.8) were

210 measured using standard spectrophotometric assay procedures described previously (Zhao and

211 Zera, 2001; Zera and Zhao, 2003), with a few modifications. Enzymes activities were measured

212 on a different group of crickets from those used to measure lipid biosynthetic rates but which 
213 were fed the same diets and raised under the same conditions. Briefly, on day 5 of adulthood,

214 after the feeding trial, $10-30 \mathrm{mg}$ of fat body were removed and homogenized in $50 \mathrm{mM} \mathrm{K}^{+}-$

215 phosphate buffer ( $\mathrm{pH} 7.4)$, containing $0.1 \% \beta$-mercaptoethanol, $5 \mathrm{mM}$

216 ethylenediaminetetraacetic acid (EDTA) and 10\% glycerol. Enzyme homogenate was further

217 diluted with homogenizing buffer but without glycerol for reasons described below. Eight $\mu \mathrm{L}$ of

218 diluted homogenate was added to $192 \mu \mathrm{L}$ of enzyme cocktail described in (Zhao and Zera,

219 2001). Change in absorbance at $340 \mathrm{~nm}$ was measured for six minutes after one min incubation

220 at $28^{\circ} \mathrm{C}$ in a Fluorostar Omega spectrophotometer. Previous studies had shown that six

221 minutes was within the "linear" period of the progress curve and that the enzyme dilutions used

222 resulted in activities that were linear with respect to enzyme concentration; both preconditions

223 are needed for steady-state kinetic analysis. Protein concentrations of homogenates were

224 measured using the Bradford protein assay with bovine serum albumen as the standard

225 (Stoscheck, 1990).

227 Statistical analyses and response surfaces. General linear models performed in R (version

228 2.15.3) were used to analyze biochemical and reproductive characteristics as a function of \%

229 carbohydrate and \% protein in the diet. In the present study, we were mainly interested in

230 assessing morph differences in response variables (e.g. \% incorporation of radiolabel into lipid,

231 enzyme specific activity, ovarian weight) as a function of diet macronutrients (\% carbohydrate

232 and \% protein). The overall difference between the LW(f) and SW morphs for a particular

233 variable was assessed by a partial F-test in which the full statistical model containing all morph

234 and macronutrient terms (main effects of morph, protein and carbohydrate, as well as eight

235 interaction terms) was compared with a reduced model without any morph terms (see below

236 and Clark et al., 2015, 2016). A significant difference between the two models was interpreted

237 as a significant overall effect of morph. Results of the analyses of the full model containing all

238 morph, protein, and carbohydrate main effects and interaction terms are reported because 
239 these results illustrate the nature of the morph differences. In one case (ovarian lipid

240 biosynthesis), no significant effect of morph was found and thus results of the reduced model

241 without morph terms are reported. This reduced analysis is included as it assesses effects of

242 macronutrients independent of morph on response variables. In these analyses, macronutrient

243 axes were standardized using the rsm package (Lenth, 2009).

244 Variation in each response variable for each morph is presented as a non-parametric

245 response surface figure. These surfaces provide a more detailed visualization of the

246 experimental data as compared with graphing the best-fitting response surface regression

247 models. These non-parametric response surface figures were generated with the thin-plate

248 splines function (Tps) from the "fields" package in R (Furrer et al., 2012), using lambda set

249 equal to 0.01 . However, response surface figures only provide a qualitative measure of morph-

250 specific variation in response variables. Thus, quantitative differences between separate morph-

251 specific response surfaces were assessed by statistical comparisons of the estimated linear and

252 quadratic terms for diet protein and carbohydrate content, as well as protein X carbohydrate

253 interaction term(s). A significant and negative quadratic term (i.e. protein $\mathrm{X}$ protein or

254 carbohydrate $\mathrm{X}$ carbohydrate) indicates that the response surface is curved in that dimension,

255 and that an intermediate amount of nutrient produces a maximal response. In contrast, if only

256 linear model terms are significant, the response surface shape is flat, and maximal and minimal

257 responses occur at edges of the response surface. Significant protein $\mathrm{X}$ carbohydrate

258 interaction terms indicate that the response surface shape is complex, and response

259 characteristics will depend on specific combinations of protein and carbohydrate for each

260 morph.

261

262 Results 
263 Means_SEMs of each response variable for each morph on each diet, which were used to

264 construct the response surfaces, are given in Appendix 2. Summary of the statistical analyses of

265 these data are given in Table 1, while the full results of analyses are given in Appendix 3.

267 Ovarian masses. At the end of the 5-day feeding trial, ovarian wet mass standardized to whole-

268 body wet mass (\%) was substantially larger for the SW morph compared with the LW(f) morph

269 over the entire protein-carbohydrate landscape [Morph means ( + SEM): LW(f): 4.4+0.3\%; SW:

270 10.3+0.5\%;_Fig 1A, B; partial F-test of models with (= full) and without (= reduced) "morph":

$271 \quad F_{6,126}=15.4, P<0.001$, Table 1, Appendix $\left.3 A\right)$. Ovarian mass differed between morphs $\left(F_{1,126}=\right.$

272 128.1, $P<0.001)$, and there was a significant morph $X$ protein interaction $\left(F_{1,126}=5.6, P=0.02\right)$.

273 This interaction was due to increasing ovarian mass in the SW morph with increasing diet

274 protein content (Fig 1B), which resulted in a significant linear protein coefficient: 22.8+6.7; $\mathrm{P}<$

2750.05 , Table 2. By contrast, the LW(f) morph exhibited smaller ovaries that were nearly invariant

276 across the entire protein-carbohydrate landscape (Fig 1A).

277

278 Whole-body lipid biosynthesis. Across all diets, percent incorporation of ${ }^{14} \mathrm{C}$-acetate into

279 whole-body lipid during the three $\mathrm{h}$ incubation period was substantially (ca. 50\%) higher in the

$280 \mathrm{LW}(\mathrm{f})$ compared to the SW morph $[\mathrm{LW}(\mathrm{f})=16.5 \pm 0.95 \%, \mathrm{~N}=53 ; \mathrm{SW}=10.9 \pm 0.90 \%, \mathrm{~N}=56$;

281 Partial F-test of full vs. reduced models: $F_{6,96}=5.74, P<0.001$; Fig. 1C, D; Table 1; Appendix 3B].

282 This is evident by the much higher incorporation values across nearly the entire protein-

283 carbohydrate landscape for the LW(f) morph (Fig 1C, D).

284 Of the two main macronutrients in the diet, carbohydrate strongly and positively affected

285 lipid biosynthesis $\left(F_{1,96}=20.6 ; P<0.0001\right.$, Appendix $\left.3 B\right)$, while protein content had no observed

286 effect. This is clearly seen in the response surface graphs (Fig 1C, 1D) in which rate of lipid

287 biosynthesis increased linearly (i.e. vertically) with dietary carbohydrate for each morph. 
In addition to these main effects, a highly significant morph $\mathrm{X}$ carbohydrate interaction on the rate of whole-body lipid biosynthesis was also observed $\left(F_{1,96}=7.24, P=0.008\right.$; Table 1 ;

290 Appendix 3B). This is evident qualitatively by the much greater increase in the rate of lipid 291 biosynthesis with increasing dietary carbohydrate in the LW(f) morph compared with the SW 292 morph (Fig 1C, D), and quantitatively by the highly significant linear coefficient for the effect of 293 carbohydrate on the response surface (Table 2$)$ for the $\operatorname{LW}(f)$ morph $(7.66 \pm 1.20, P<0.05)$ but

294 not the SW morph $(1.62 \pm 1.27$, ns). Finally, there was a significant protein X carbohydrate 295 interaction for the SW but not the LW(f) morph (protein X carbohydrate coefficient for the SW 296 morph $=-4.72+2.35, \mathrm{P}<0.05$, Table 2$)$. This was evident by the increased rate of lipid 297 biosynthesis on two opposing edges of the response surface corresponding to high protein and 298 low carbohydrate diets, but vice versa for the SW morph (Fig 1C, D). In summary, there was an 299 overall higher rate of whole-body lipid biosynthesis for the LW(f) compared to the SW morph 300 across the entire protein-carbohydrate landscape, and the magnitude of the difference between 301 the morphs also varied across the landscape mainly as a function of carbohydrate content of the 302 diet. Near the very bottom of the response surface graph (lowest carbohydrate diets), rate of 303 lipid biosynthesis was the lowest for each morph and the least different between morphs.

305 Somatic lipid allocation. Radiobiosynthesized lipid found in the soma (whole body minus 306 ovaries) was significantly higher in the $\operatorname{LW}(\mathrm{f})$ morph $(14.9 \pm 0.80 \% ; \mathrm{N}=68)$ compared with the 307 SW morph $\left(9.10 \pm 0.73 ; \mathrm{N}=69\right.$; partial F-test of full and reduced models: $\mathrm{F}_{6,124}=8.23, \mathrm{P}<0.001$; 308 Table 1; Appendix 3.C; Fig 2A, B). As was the case for total lipid biosynthesis, carbohydrate 309 content of the diet also affected the amount of radiolabel incorporated into somatic lipid $310\left(F_{1,124}=35.6, P<0.0001\right)$, and there was a strong morph $X$ carbohydrate interaction $\left(F_{1,124}=11.13\right.$, $311 P=0.001)$. Significant linear coefficients for the effect of carbohydrate on the separate morph 312 response surfaces were observed for both the $\operatorname{LW}(f)(8.34 \pm 1.65, P<0.05)$ and SW morphs $313(2.26 \pm 1.04, \mathrm{P}<0.05$; Table 3$)$ indicating a positive effect of carbohydrate on the amount of 
314 somatic lipid produced. The effect of carbohydrate was four times stronger in the LW(f) morph,

315 thus giving rise to the morph $\mathrm{X}$ carbohydrate interaction, similar to the situation mentioned

316 above for whole body lipid biosynthetic rate. Finally, as also was the case for whole-body lipid

317 biosynthesis, there was a significant protein $\mathrm{X}$ carbohydrate coefficient for the SW morph (-

$3184.88 \pm 1.99, \mathrm{P}<0.05)$, but not for the $\operatorname{LW}(\mathrm{f})$ morph $(1.71 \pm 3.09, \mathrm{~ns}$; Table 3$)$. This was due to the

319 increased amount of biosynthesized somatic lipid produced in SW, but not LW(f) morphs, fed on

320 diets at the edges of the response surface (Fig. 2A, B).

321

322 Ovarian lipid allocation. In contrast to the situation for whole-body or somatic lipid, amount of

323 newly biosynthesized lipid found in the ovaries was higher in SW $(1.63 \pm 0.12 \%, N=53)$

324 compared with $\operatorname{LW}(f)$ females $(0.92 \pm 0.13 \% ; N=56)$ across the protein-carbohydrate landscape

325 (Fig 2B, C; Partial F-test of full and reduced models: $F_{6,97}=3.47, P=0.0014$; Table 1; Appendix

326 3D). Biosynthesized lipid in the ovaries was uniform across the protein-carbohydrate landscape

327 in the LW(f) morph (Fig 2C; no significant linear or quadratic response surface terms for protein,

328 carbohydrate or interactions; Table 3). In the SW morph, a greater amount of biosynthesized

329 lipid in the ovaries was associated with increasing protein content of the diet (Fig 2D; linear

330 coefficient for protein: $0.56 \pm 0.25, P<0.05$, Table 3$)$. Rates also increased with increasing

331 carbohydrate content of the diet, although the linear carbohydrate coefficient was non-significant

$332(0.40 \pm 0.25, \mathrm{~ns})$ and there was a significant protein $\mathrm{X}$ carbohydrate interaction in the SW morph

333 (Fig 2D; protein $X$ carbohydrate coefficient: $-0.96+0.45, P<0.05$, Table 3 ). When ovarian mass

334 was used as a covariate in an ANCOVA, no significant effect of morph or diet was observed on

335 the amount of biosynthesized lipid in the ovary (partial F-test of full and reduced models: $F_{6,96}=$

$3360.47, \mathrm{~ns})$. This indicates that the greater amount of biosynthesized lipid in the ovaries of SW

337 females appears to be associated entirely with the larger size of the ovaries in that morph. 

compartments. Over the entire protein-carbohydrate landscape, the percentage of whole-body

341 radiolabeled lipid found in the somatic versus ovarian compartments was significantly higher in

342 the LW(f) morph $(93.0 \pm 1.01 \% ; N=53)$ compared with the SW morph $(84.0 \pm 0.96 \% ; N=56$;

343 Partial F-test: $F_{6,97}=7.71, P<0.001$; Fig 2E, F; Table 1; Appendix 3E). Across morphs, there was

344 no overall effect of either major dietary macronutrient on the degree of newly biosynthesized

345 lipid allocated to the somatic vs. ovarian body compartments $(P>0.1$ for either protein or

346 carbohydrate, Table 3; Appendix 3E). However, within the LW(f) morph there was a significant

347 linear coefficient for carbohydrate $(0.028+0.012, P<0.05$; Table 3$)$ compared to a non-significant

348 coefficient in the SW morph (Table 3). Inspection of the response surfaces (Fig 2E, F) indicates

349 that this was due to a significantly greater allocation of biosynthesized lipid to the soma with

350 increasing carbohydrate in the diet.

352 NADP+-IDH specific activity. Like the rate of whole-body or somatic lipid biosynthesis, NADP ${ }^{+}-$

353 IDH specific activity of the fat body was significantly higher in the LW(f) morph compared to the

354 SW morph across the entire protein-carbohydrate landscape (morph means $( \pm S E M)$ : $L W(f)=$

$355 \quad 233.1 \pm 11.2, \mathrm{SW}=169.2 \pm 7.1 \mathrm{nmol} / \mathrm{min} / \mathrm{mg}$ protein; Fig $3 \mathrm{~A}, \mathrm{~B} ;$ Partial F-test: $\mathrm{F}_{6,112}=6.35$,

$356 \mathrm{P}<0.001$; Table 1; Appendix 3F). For the LW(f) morph, NADP ${ }^{+}$-IDH specific activity was

357 essentially uniform across the response surface (Fig $3 \mathrm{~A}$ ), with no significant linear or quadratic

358 terms for protein or carbohydrate (Table 4). By contrast, for the SW morph, NADP ${ }^{+}$-IDH specific

359 activity rose linearly with carbohydrate content (Fig 3B; carbohydrate linear coefficient $=$

$360 \quad 0.39 \pm 0.14, P<0.05$, Table 4$)$ and there was a significant quadratic coefficient for carbohydrate

$361(0.74 \underline{+0.26, P<0.05) .}$

363 ACL specific activity. ACL also exhibited a significantly higher fat body specific activity in the

$364 \mathrm{LW}(\mathrm{f})$ morph compared to the SW morph (Partial F-test: $F_{6,138}=6.35, \mathrm{P}<0.001$; Table 1; 
Appendix $3 G$ ) across the entire protein-carbohydrate landscape (morph means( \pm SEM): LW(f) 33.8+2.5, SW = 23.8+2.1Fig 3C, D). However, no significant main effect of morph was observed

$367\left(F_{1,138}=0.23, P=0.63 ;\right.$ Table 1; Fig 3C, D). Neither did protein nor carbohydrate exhibit 368 significant main effects on ACL specific activity $(P>0.3$, Table 1; Appendix $3 G)$. However, there 369 were numerous (5) significant interaction terms involving various combinations of morph,

370 protein, or carbohydrate (Table 1; Appendix $3 G$ ), for example, morph $X$ protein $\left[F_{1,138}=10.4, P\right.$ $371=0.002]$ and morph $X$ carbohydrate $X$ protein $\left(F_{1,138}=10.3, P=0.002\right)$. This indicates complex 372 morph-specific response surfaces in which multiple peaks differed in location and height in a 373 morph-specific manner (see Figs 3C, D). For example, the LW(f) morph exhibited higher peaks

374 of ACL specific activity in the two quadrants of the response surface comprising (1) high-protein, 375 low carbohydrate (e.g. diet p28:c14) and (2) high carbohydrate, low protein (p8:c34). These 376 were not seen in the SW landscape. By contrast, the ACL specific activity response surface of 377 the SW morph exhibited peaks in the other two quadrants (e.g. p9:c12 and p16.25:c35.25) that 378 were not seen in the LW landscape. These qualitative differences were manifest quantitatively 379 as significant coefficients for protein $\mathrm{X}$ protein $(3.27 \pm 1.23, \mathrm{P}<0.05)$ and protein $\mathrm{X}$ carbohydrate $380(-4.73+1.24, \mathrm{P}<0.05)$ in the $\mathrm{LW}(\mathrm{f})$ but not SW surfaces (Table 4). Finally, a morph-specific 381 effect of protein on ACL specific activity is evidenced by the significant linear protein coefficient 382 in the SW morph $(-1.43 \pm 0.59 \mathrm{P}<0.05$; Table 4) that was not significant in the LW(f) morph.

\section{Discussion}

385 The wing-polymorphic cricket, Gryllus firmus, has emerged as an important model species in 386 studies of the biochemical basis of life history variation and trade-offs in outbred populations 387 (e.g., Zhao and Zera, 2002; Zera, 2005; Zera and Zhao, 2003, 2006; Zera and Harshman, 2009, 388 2011; Schilder et al., 2011). Although previous investigations documented large magnitude 389 differences between morphs in many aspects of lipid metabolism (see Introduction), the 390 generality of these results was unclear because experiments were mainly conducted on a single 
391 artificial diet that contained $20 \%$ protein and $27 \%$ carbohydrate. In particular, prior experiments

392 could not assess the extent to which the morph-differences in lipid metabolism are dependent

393 upon diet protein-carbohydrate content. In the current study we examined how diet influenced

394 morph-differences in lipid metabolism by conducting radiotracer and enzymological

395 investigations across 13 ecologically relevant diets that differed in their protein-carbohydrate

396 content. Our results confirm and extend key findings of previous biochemical studies, but

397 require reevaluation of others.

399 Morph differences in lipid biosynthesis across the protein-carbohydrate landscape. One

400 important finding of the present study was the overall greater rate of whole-body lipid

401 biosynthesis in the $\mathrm{LW}(\mathrm{f})$ compared to the SW morph across the entire protein-carbohydrate

402 landscape (Figs. 1C, D). The only local exception to this general pattern was the nearly equal

403 and low biosynthetic rates of the morphs on very low carbohydrate and protein diets (bottom of

404 Figs 1C, D). Thus, the greater rate of lipid biosynthesis of LW(f) vs. SW G. firmus reported

405 previously on a limited number of diets (Zhao and Zera, 2002; Zera, 2005), appears to be a

406 general feature of the polymorphism across a wide range of diets. Although crickets are

407 omnivorous, little is known about the exact composition of cricket diets in the field. However,

408 diets used in the present study cover the range of protein and carbohydrate levels found in

409 plants (high carbohydrate, low protein - diets a, b, d and g; see Appendix 1 and Figure 1A) and

410 animals (lower carbohydrate, higher protein - diets c, i and j). Thus, findings of biochemical and

411 physiological studies of morph-specific lipogenesis conducted in the laboratory on these diets

412 can likely be extrapolated to field environments.

413 De novo fatty acid and glyceride biosynthesis primarily occur in the fat body, after which

414 newly biosynthesized lipid is either stored in this organ, mainly in the form of triglyceride, or is

415 transported to other organs (e.g. as a component of vitellogenin, transported to the ovaries;

416 Candy, 1985; Ziegler, 1997; Hagedorn and Kunkel, 1979; Klowden, 2002). Because we 
417 separately quantified the amount of newly biosynthesized lipid in the soma (body minus ovaries)

418 or ovaries, we could determine the amount that was allocated to each of these body

419 compartments across the protein-carbohydrate landscape. This is important because a central

420 issue in life history physiology concerns the relative allocation of nutrients to somatic vs.

421 reproductive functions (Zera and Harshman, 2001; Harshman and Zera, 2007; Flatt and

422 Heyland, 2011). Lipid is an important energy-rich component of eggs (ca. 25\% dry weight of $G$.

423 firmus eggs; Zera et al., 1994) as well as being an important somatic energy reserve. While a

424 number of studies have quantified whole-body lipid in a life history context, the reproductive

425 versus somatic components have not always been separated (Zera and Harshman, 2001). This,

426 in turn, has resulted in overestimates of nutrient allocation to the soma and underestimates in

427 allocation to reproduction, in cases in which whole body lipid has been equated with energetic

428 investment to somatic functions (Zera and Harshman, 2001; Zhao and Zera, 2002; Zera and

429 Harshman, 2011).

430 In both morphs, the majority of total biosynthesized lipid was found in the soma (85-95\%;

431 Fig 2E, F; see below), and the amount of somatic lipid was significantly greater for the LW(f)

432 compared to the SW morph (Figs. 2E, F). This difference was due not only to the greater rate of

433 whole-body lipid biosynthesis (Fig 1C, D), but also to the greater proportion of whole-body lipid

434 biosynthesis allocated to the soma versus ovaries in $\mathrm{LW}(\mathrm{f})$ females $(93.1 \pm 0.7 \%)$ vs. SW

435 females (SW: 84.0+1.2\%; Fig. 2E, F). In other words, there was a trade-off between the morphs

436 in the extent to which newly biosynthesized lipid was preferentially allocated to the soma versus

437 ovaries over the entire protein-carbohydrate landscape, similar to the morph-specific trade-off

438 observed previously on the standard laboratory diet (Zhao and Zera, 2002). Both elevated lipid

439 biosynthesis and greater allocation to the soma by LW(f) females are likely important

440 contributors to the elevated total somatic lipid content in LW(f) compared to SW females across

441 the entire protein-carbohydrate landscape (Clark et al., 2015; Zera, Clark and Behmer,

442 unpublished data). This current finding suggests that overall morph-specific differences in lipid 
443 levels, as reported in Clark et al. (2015), can largely be explained by morph-specific differences

444 in lipid biosynthesis, and not just differences in the amount of carbohydrate eaten. However, the 445 importance of morph-specific differences in lipid oxidation have yet to be taken into account.

446 In several cases, diet appeared to significantly affect the magnitude of the morph

447 difference in biosynthesized somatic lipid (see Results). Most important was the greater positive 448 effect of carbohydrate on lipid production in LW(f) compared with SW G. firmus (Fig 1C, D). This 449 was associated with a greater relative increase of whole-body lipogenesis in LW(f) compared to $450 \mathrm{SW}$ in the high carbohydrate region of the protein-carbohydrate landscape. In addition, the 451 relative amount of lipid allocated to the soma versus the ovaries (i.e. the magnitude of the 452 somatic-ovarian trade-off) was also greater in LW(f) vs SW females on high carbohydrate diets 453 (Fig 2E, F). The positive effect of dietary carbohydrate on lipid biosynthesis in animals has been 454 well-known for decades (MacDonald, 1966; Hori and Nakasone, 1971; Geer and Perille, 1977;

455 Greener and van Golde, 1977; Goodridge, 1987; Girard et al., 1997), as has the influence of 456 genotype of laboratory stocks on the degree to which lipogenesis is affected by dietary 457 carbohydrate (Berdanier, Tobin and Devore, 1979; Geer and Laurie-Ahlberg, 1984; Reed et al., 458 2010; Musselman et al., 2013). A unique aspect of the present study is the demonstration that 459 diet protein-carbohydrate profile appears to strongly influence the magnitude of lipid 460 biosynthesis differences between morphs of $G$. firmus. Across the landscape, this could be due 461 to several factors that may act in concert. The LW(f) morph might be inherently programmed to 462 divert more carbohydrate to lipid on high carbohydrate diets even when an equal amount of 463 carbohydrate is consumed by the morphs (i.e. pathway enzymes in the LW(f) morph might be 464 more sensitive to carbohydrate or carbohydrate derivatives). Alternatively, greater consumption 465 by the LW(f) morph on high carbohydrate diets might provide more substrate for lipogenesis. A 466 previous feeding study demonstrated greater food consumption for the LW(f) morph compared 467 with the SW morph on some but not all diets in the high carbohydrate region of the nutrient 468 landscape (see Fig. 2C of Clark et al. 2015). 
The much smaller effect of carbohydrate on lipid allocation to the soma in the SW

470 morph might be due to a constraint in the degree to which lipid can be stored in the soma

471 relative to the $\operatorname{LW}(\mathrm{f})$ morph. On the standard laboratory diet, SW females, compared to $L W(f)$

472 females, have about half the amount of fat body (Zera and Zhao, 2003; and unpublished data),

473 possibly due to the much larger ovaries in the SW morph coupled with limited thoracic and

474 abdominal space. Increased ovarian mass in SW females likely occurs at the expense of fat

475 body mass, thus limiting somatic lipid storage capacity. Recent studies indicate that the ability to

476 convert carbohydrate into lipid is not only important with respect to producing somatic and

477 reproductive energy reserves, it also serves as a mechanism by which organisms (including

478 insects such as Drosophila) tolerate high carbohydrate diets by converting carbohydrate into

479 lipid, because it reduces glucose concentration in the blood (Musselman et al., 2013). An

480 emerging concept in human diabetes research is that fat accumulation per se is less harmful

481 than the production of free fatty acids and their derivatives, which occurs when the capacity of

482 the organism to convert fatty acids to triglyceride stores in the fat body is exceeded (termed

483 "lipotoxicity; Brookheart et al., 2009; Neuschwander-Tetri, 2010). This suggests that, if the SW

484 morph of $G$. firmus has reduced ability to convert dietary carbohydrate into stored triglyceride,

485 this morph might be more sensitive to the deleterious effect of a high carbohydrate diet than the

$486 \mathrm{LW}(\mathrm{f})$ morph. Consistent with this hypothesis, preliminary data indicate that glucose oxidation is

487 higher in SW than in LW(f) females across the protein-carbohydrate landscape in these LW(f)

488 and SW populations (Zera, Clark, Behmer, unpubl data). In addition a greater proportion of

489 carbohydrates is oxidized on high carbohydrate diets by both morphs (Clark et al., 2016).

490 SW females allocated a greater amount of biosythesized lipid than LW(f) females to the

491 ovaries over the entire protein-carbohydrate landscape (Fig 2C, D); this was expected because

492 SW females have larger ovaries. However, the morph-specific pattern of lipid allocation to the

493 ovaries differed from that in the soma in some respects. Ovarian allocation was relatively low

494 and uniform across the protein-carbohydrate landscape in LW(f) females (Fig 2C). By contrast, 
495 SW females increased lipid allocation to the ovaries as a function of dietary protein, and to a 496 lesser degree (and non-significantly) as a function of dietary carbohydrate. Ovarian mass was

497 substantially higher in SW versus LW(f) females across the entire protein-carbohydrate

498 landscape, but especially so in the high protein region (Fig 2D). Thus, the greater allocation of

499 lipid to the ovaries on high protein diets in SW females might be a requirement to accommodate

500 greater growth of eggs on those diets, because eggs have a high lipid content (ca. $25 \%$ dry

501 mass; Zera et al., 1984).

502

503 Morph-specific differences in enzyme activities across the protein-carbohydrate

504 landscape. In previous studies on the standard laboratory diet, a straightforward relationship 505 was observed between specific activities of five lipogenic enzymes, including fat body NADP ${ }^{+}$

$506 \mathrm{IDH}$ and ACL, and rate of lipid biosynthesis. Specific activities of all lipogenic enzymes as well

507 as the rate of lipid biosynthesis were elevated to a similar degree in the LW(f) compared to the

508 SW morph, and strongly co-segregated with the LW(f) morph in crosses between the LW(f) and

509 SW stocks (Zera and Zhao, 2003). This result suggested that morphs might differ genetically in

510 a few regulators that co-ordinate many aspects of lipid metabolism (Zera and Zhao, 2003). In

511 the present study, undertaken across a wider range of diets, less concordant patterns were

512 observed between specific activities of lipogenic enzymes and rate of lipid biosynthesis.

513 As in previous studies, specific activities for NADP ${ }^{+}$-IDH were higher in LW(f) than SW

514 females across the entire protein-carbohydrate landscape (Fig 3A, B). This is consistent with

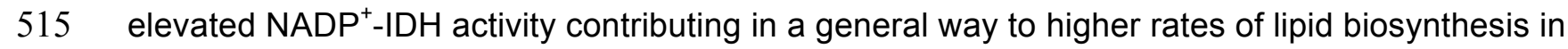

$516 \mathrm{LW}(\mathrm{f})$ females across all diets (Fig 1C, D). Nevertheless, on the highest carbohydrate diets,

517 activities of this enzyme were similar in LW(f) and SW crickets, due to the positive effect of

518 dietary carbohydrate on $\mathrm{NADP}^{+}$-IDH activity in SW, but not LW(f) females (Figs 3A, B). These

519 patterns did not match the much greater rate of lipid biosynthesis in the LW(f) morph on the high 
520 carbohydrate diets, nor the positive effect of carbohydrate on lipid biosynthesis in LW(f) but not 521 SW females (see above).

522 One possible explanation for this discordant pattern is that NADP ${ }^{+}$-IDH might be a less

523 important contributor to morph-specific differences in lipid biosynthesis in the high -

524 carbohydrate region of the nutrient landscape. A variety of enzymes other than NADP ${ }^{+}$-IDH

525 contribute NADPH for lipid biosynthesis (e.g. malic enzyme (ME), glucose-6-phosphate

526 dehydrogenase (G-6-PDH), and 6-phosphogluconate dehydrogenase(6-PGDH)). In $D$.

527 melanogaster, the pentose-shunt enzymes, G-6-PDH and G-PGDH appear to be more

528 important than NADP ${ }^{+}-$IDH in lipogensis on high-carbohydrate diets (Geer et al., 1978). More

529 recently, Rzezniczak and Merrit (2012) reported complex interactions among NADPH-producing

530 enzymes in D. melanogaster in different environments. This further suggests that individual

$531 \mathrm{NADP}^{+}$-producing enzymes, such as NADP ${ }^{+}$-IDH in G. firmus, may have unexpected

532 environment-contingent roles in metabolism.

533 ATP-citrate lyase (ACL), the other lipogenic enzyme investigated, showed particularly

534 complex patterns of fat-body specific activities in which morph-specific differences in activity of

535 this enzyme were strongly contingent upon diet (Figs 3C, D). On the one hand, the significantly

536 higher ACL specific activity in LW(f) vs. SW females fed high protein-low carbohydrate diets

537 suggests that this enzyme might be especially important in contributing to the greater lipid

538 biosynthesis in LW(f) compared to SW females on this part of the nutrient landscape.

539 Surprisingly, like NADP ${ }^{+}-\mathrm{IDH}, \mathrm{ACL}$ specific activity was also not correlated with the highest rates

540 of lipid biosynthesis in the LW(f) morph, which occurred on the highest carbohydrate diets.

541 Results of studies on $\mathrm{NADP}^{+}-\mathrm{IDH}$ and $\mathrm{ACL}$ specific activities to date suggest a complex shifting

542 of importance of various enzymes in contributing to morph-specific differences in lipid

543 biosynthesis across the nutrient landscape. This picture contrasts with the more simplified

544 picture previously proposed regarding the role of variation in lipogenic enzyme activities and 
545 lipid biosynthesis (Zera and Zhao, 2003; see above), and is more consistent with the view of 546 strong environment-dependent interactions of enzyme networks (Rzezniczak and Merritt, 2012).

Another intriguing possibility is that variation in activities of lipogenic enzymes other than

548 those involved in fatty acid biosynthesis might be more important with respect to causing morph-

549 specific differences in lipogenesis across the nutrient landscape. Most newly biosythesized lipid

550 in G. firmus is triglyceride (85\%; Zhao and Zera, 2002; and unpublished), which is produced by

551 enzymes of the glyceride pathway which link fatty acids to the glycerol-3-phosphate backbone.

552 The glyceride pathway functions downstream of the pathway of de novo fatty acid biosynthesis

553 in which acetyl-CoA is converted to fatty acid. Thus far, all enzymes whose activities have been

554 measured in morphs of G. firmus in the context of lipid biosynthesis (e.g. ACL and NADP ${ }^{+}$IDH

555 in the present study) are involved in fatty acid biosynthesis. However, a recent transcriptome

556 study identified morph differences in gene expression of some enzymes of glyceride

557 biosynthesis, as well as enzymes involved in modification of fatty acids such as elongases,

558 reductases, and desaturases, as being much larger than those of genes encoding enzymes of

559 de novo fatty acid biosynthesis (Vellichirammal et al., 2014). This suggests that enzymes

560 involved in lipogenesis, that are downstream from de novo fatty acid biosynthesis, may play a

561 more important role in regulating morph differences in overall triglyceride production.

562 Finally, both NADP+-IDH and ACL are involved in metabolic roles other than

563 lipogenesis. For example, in many organisms undergoing oxidative stress, NADP ${ }^{+}$-IDH

564 produces NADPH, which is subsequently used to produce antioxidants such as reduced

565 glutathione (Lee et al., 2002; Rzezniczak and Merritt. 2012; Dey et al., 2016). Thus, it is

566 conceivable that the unexpected greater increase in NADP ${ }^{+}-I D H$ in the SW morph on high

567 carbohydrate diets, mentioned above, may combat increased oxidative stress, which often

568 accompanies lipotoxicity (Brookheart et al., 2009). In the same vein, high ACL activity occurs in

569 some organs for purposes other than increased lipogenesis, for example, to metabolize citrate

570 as part of the mechanism of hormonal secretion (Guay et al., 2007). There are many possible 
571 reasons for the unexpected morph- and diet-specific patterns of NADP+-IDH and ACL activities.

572 These results highlight our ignorance of the specific roles played by enzymes in morph specific-

573 metabolic adaptation to nutrient heterogeneity.

574

575 
576 Acknowledgements

577

$578 \quad$ Funding

579 This work was supported by the National Science Foundation [grant number IOS-1121960 to

580 S.T.B. and IOS-1122075 to A.J.Z.].

581

582 


\section{References}

584

585

586

587

588

589

590

591

592

593

594

595

596

597

598

599

600

601

602

603

604

605

606

607

608

609

610

611

612

613

614

615

616

617

618

619

620

621

622

623

624

625

626

627

628

629

630

631

632

Behmer, S.T. 2009. Insect herbivore nutrient regulation. Annu. Rev. Entomol. 54:165-187.

Berdanier, C.D., Tobin, R.B., Devore, A. 1979. Effect of age, strain, and dietary carbohydrate on the hepatic metabolism of male rats. J. Nutr. 109:261-271.

Boggs, C.L. 2009. Understanding insect life histories and senescence through a resource allocation lens. Funct. Ecol. 23:27-37.

Brookheart, R.T., Michel, C.I., Schaffer, J.E. 2009. As a matter of fat. Cell Metab. 10:9-12.

Candy, D.J. 1985. Intermediary metabolism. Pp. 1-41 in G. A. Kerkut and L. I. Gilbert, eds. Comprehensive Insect Physiology Biochemistry and Pharmacology. Pergamon, Oxford.

Clark, R.M., Zera, A.J., Behmer, S.T. 2015. Nutritional physiology of life-history trade-offs: how food protein-carbohydrate content influences life-history traits in the wing-polymorphic cricket Gryllus firmus. J. Exp. Biol. 218: 1-11.

Clark, R.M., Zera, A.J., Behmer, S.T. 2016. Metabolic rate is canalized in the face of variable life history and nutritional environment. Funct. Ecol. 30: 922-931.

Dey, S., Sidor, A., O'Rourke, B. 2016. Compartment-specific control of reactive oxygen species scavenging by antioxidant pathway enzymes. J. Biol. Chem. 291:11185-11192.

Flatt, T., Heyland, A. 2011. Mechanisms of Life History Evolution. Oxford University Press, Oxford.

Furrer, R., Nychka, D., Sain, S. 2012. fields: tools for spatial data (Version 6.7). Retrieved from http://CRAN.R-project.org/package=fields

Geer, B.W., Kamiak, S.N., Kidd, K.R., Nishimura, R.A., Yemm, S.J.. 1976. Regulation of the oxidative NADP-enzyme tissue levels in Drosophila melanogaster. I. Modulation by dietary carbohydrate and lipid. J. Exp. Zool. 195:15-32.

Geer, B.W., Perille, T.J. 1977. Effects of deitary sucrose and environmental temperature on fatty acid synthesis in Drosophila melanogaster. Insect Biochem. 7:371-379.

Geer, B.W., Woodward, C.G., Marshall, S.D. 1978. Regulation of the oxidative NADP-enzyme tissue levels in Drosophila melanogaster. II The biochemical basis of dietary carbohydrate and D-glycerate modulation. J. Exp. Zool. 203:391-402.

Geer, B.W., Laurie-Ahlberg, C.C. 1984. Genetic variation in the dietary sucrose modulation of enzyme activities in Drosophila melanogaster. Genet. Res. Camb. 43:307-321.

Girard, J., Ferre, P., Foufelle, F. 1997. Mechanisms by which carbohydrates regulate expression of genes for glycolytic and lipogenic enzymes. Annu. Rev. Nutr. 17:325-352.

Goodridge, A.G. 1987. Dietary regulation of gene expression: Enzymes involved in carbohydrate and lipid metabolism. Annu Rev. Nutr. 7:157-185. 
633

634

635

636

637

638

639

640

641

642

643

644

645

646

647

648

649

650

651

652

653

654

655

656

657

658

659

660

661

662

663

664

665

666

667

668

669

670

671

672

673

674

675

676

677

678

679

680

681

682

683

Groener, J.E.M., van Golde, L.M.G. 1977. Effect of fasting and feeding a high-sucrose, fat-free diet on the synthesis of hepatic glycerolipids in vivo and in isolated hepatocytes. Biochem. Biophys. Acta 487:105-114.

Guay, C., Madiraju, S.R., Aumais, J. E., Prentki, M. 2007. A role for ATP-citrate lyase, malic enzyme and pyruvate/citrate cycling in glucose-induced insulin secretion. J. Biol. Chem. 282:3567-3565.

Guerra, P.A. 2011. Evaluating the life history trade-off between dispersal capability and reproduction in wing dimorphic insects: a meta-analysis. Biol. Rev. 86:813-835.

Hagedorn, H.H., Kunkel, J.G. 1979. Vitellogenin and vitellin in insects. Annu. Rev. Entomol. 24: 475-505.

Harrison, R.G. 1980. Dispersal polymorphisms in insects. Annu. Rev. Ecol. Syst. 11:95-118.

Harshman, L.G., Zera, A.J. 2007. The cost of reproduction: the devil in the details. Trend. Ecol. Evol. 22:80-86.

Hochachka, P.W., Somero, G.N. 2002. Biochemical Adaptation. Mechanism and Process in Physiological Evolution. Oxford University Press, Oxford.

Hori, Y., Nakasone, S. 1971. Effects of the levels of fatty acids and carbohydrates in a diet on the biosynthesis of fatty acids in the larvae of the silkworm, Bombyx mori. J. Insect Physiol. 17:1441-1450.

Klowden, M. 2002. Physiological Systems in Insects. Academic Press, Amsterdam.

Lee, S.M., Koh, H-J., Park, D-C., Song, B.J., Huh, T-L., Park, J-W. 2002. Cytosolic NADP+dependent isocitrate dehydrogenase status modulates oxidative damage to cells. Free Radical Biol. Med. 32:1185-1196.

Lee, K. P., Simpson, S.J., Clissold, F.J., Brooks, R., Ballard, J.W.O., Taylor, P.W., Soran, N., Raubenheimer D. 2008. Lifespan and reproduction in Drosophila: New insights from nutritional geometry. Proc. Natl. Acad. Sci. USA 105:2498-2503.

Lenth, R.V. 2009. Response-surface methods in R, using rsm. J. Stat. Soft. 32:1-17.

MacDonald, I. 1966. Lipid responses to dietary carbohydrates. Advan. Lipid Res. 4:39-67.

Musselman, L.P., Fink, J.L., Vankatesh, P., Patterson, B.W., Okunade, A.L., Maier, E., Brent, M.R., Turk, J., Baranski, T.J.. 2013. Role of fat body lipogenesis in protection against the effects of caloric overload in Drosophila. J. Biol. Chem. 288:8028-8042.

Neuschwander-Tetri, B.A. 2010. Nontriglyceride hepatic lipotxicity: the new paradigm for the pathogenesis of NASH. Curr. Gastroenterol. Rep. 12:49-56.

Reed, L. K., Williams, S., Springston, M., Brown, J., Freeman, DesRoches, K.C.E., Sokolowski, M.B., Gibson, G.. 2010. Genotype-by-diet interactions drive metabolic phenotypic variation in Drosophila melanogaster. Genetics 185:1009-1019. 
684

685

686

687

688

689

690

691

692

693

694

695

696

697

698

699

700

701

702

703

704

705

706

707

708

709

710

711

712

713

714

715

716

717

718

719

720

721

722

723

724

725

726

727

728

729

730

731

732

733

734

Rzezniczak, T.Z., Merritt, T.J. 2012. Interactions of NADP-reducing enzymes across varying environmental conditions: A model of biological complexity. Genes, Genomes, Genetics 2:1613-1623.

Schilder, R.J., Zera, A.J., Black, C., Hoidel, M., Wehrkamp, C. 2011. The biochemical basis of life history adaptation: Molecular/enzymological causes of $\mathrm{NADP}^{+}$-isocitrate dehydrogenase activity differences between morphs of Gryllus firmus that differ in lipid biosynthesis and life history. Molec. Biol. Evol. 28:3381-3393.

Simpson, S.J., Raubenheimer, D. 1999. Assuaging nutritional complexity: A geometrical approach. Proc. Nutr. Sci. 58: 779-789.

Simpson, S.J., Raubenheimer, D. 2012. The Nature of Nutrition. A unifying framework from animal adaptation to human obesity. Princeton University Press, Princeton

Stoscheck, C.M. 1990. Quantitation of protein. Meth. Enzymol. 182:60-63.

van Noordwijk, A.J., de Jong, G. 1986. Acquistion and allocation of resources: their influence on variation in life history tactics. Am. Nat. 128:137-142.

Vellichirammal, N., Zera, A.J., Schilder, R.J., Wehrkamp, C., Riethoven, J.-J.M., Brisson, J.A.. 2014. De novo transcriptome assembly from fat body and flight muscle trascripts to identify morph-specific gene expression in Gryllus firmus. PLoS ONE 9: e82129.

Zera, A.J. 2009. Wing polymorphism in Gryllus (Orthoptera:Gryllidae): Proximate endocrine, energetic and biochemical bases underlying morph specializations for flight vs. reproduction. Pp. 609-653 in, T. Ananthakrishnan and D. Whitman, eds. Phenotypic Plasticity in Insects: Mechanisms and Consequences. Science Publishers, Enfield (NH).

Zera, A.J. 2005. Intermediary metabolism and life history trade-offs: Lipid metabolism in lines of the wing-polymorphic cricket, Gryllus firmus, selected for flight capability vs. early age reproduction. Integr. Comp. Biol. 45:511-524.

Zera, A.J., Brisson, J.A. 2012. Quantitative, physiological, and molecular genetics of dispersal and migration. Pp. 63-82 in J. Colbert, M. Baguette, T. Benton and J. Bullock, eds. DIspersal Ecology and Evolution. Oxford Univeristy Press, Oxford.

Zera, A.J., Denno, R.F. 1997. Physiology and ecology of dispersal polymorphism in insects. Annu. Rev. Entomol. 42:207-231.

Zera, A.J., Harshman, L.G. 2001. Physiology of life history trade-offs in animals. Annu. Rev. Ecol. Syst. 32:95-126.

Zera, A.J., Harshman, L.G.. 2011. Intermediary metabolism and the biochemical-molecular basis of life history variation and trade-offs in two insect models. Pp. 311-328 in T. Flatt and A. Heyland, eds. Mechanisms of Life History Evolution. Oxford University Press, Oxford.

Zera, A.J., Larsen, A.. 2001. The metabolic basis of life history variation: Genetic and phenotypic differences in lipid reserves among life history morphs of the wingpolymorphic cricket, Gryllus firmus. J. Insect Physiol. 47:1147-1160. 
Zera, A. J., Mole, S., Rokke, K. 1994. Lipid, carbohydrate and nitrogen content of long- and short-winged Gryllus firmus: Implications for the physiological cost of flight capability. J. Insect Physiol. 40:1037-1044.

Zera, A.J., Zhao, Z. 2003. Life-history evolution and the microevolution of intermediary metabolism: activities of lipid-metabolizing enzymes in life-history morphs of a wingdimorphic cricket. Evolution 57:568-596.

Zera, A.J., Zhao, Z. 2006. Intermediary metabolism and life-history trade-offs: Differential metabolism of amino acids underlies the dispersal-reproduction trade-off in a wingpolymorphic cricket. Am. Nat. 167:889-900.

Zhao, Z., Zera, A.J. 2001. Enzymological and radiotracer studies of lipid metabolism in the flight-capable and flightless morphs of the wing-polymorphic cricket, Gryllus firmus. J. Insect Physiol. 47:1337-1347.

Zhao, Z., Zera, A.J. 2002. Differential lipid biosynthesis underlies a tradeoff between reproduction and flight capability in a wing-polymorphic cricket. Proc. Natl. Acad. of Sci. USA 99:16829-16834.

Ziegler, R. 1997. Lipid synthesis by ovaries and fat body of Aedes aegypti (Diptera: Culicidae). Eur. J. Entomol. 94:385-391. 


\section{Figure legends}

Figure 1. Response surfaces of standardized ovarian masses and whole-body lipid biosynthesis for both morphs. Data (means+SEM) for the LW(f) morphs are shown in panels $(A)$ and $(C)$; SW morphs are shown in panels $(B)$ and $(D)$. Measurements were made on day- 5 of adulthood in BK-2 LW(f) and SW selected lines. Standardized ovarian masses were calculated as ovarian wet mass divided by whole-body wet mass. Response surfaces of absolute ovarian mass are virtually identical to those of standardized ovarian mass. Rate of whole body lipid biosynthesis = percentage of injected radiolabeled ${ }^{14} \mathrm{C}-\mathrm{Na}$-acetate incorporated into total lipid over a four hour incubation period. See Methods for additional details.

Figure 2. Response surfaces of biosynthesized lipid allocated to the soma, to the ovaries, and as a proportion allocated to the soma (whole body minus ovaries). Data (means+SEM) for $L W(f)$ morphs are shown in panels $(A),(C)$ and $(E)$; SW morphs are shown in panels $(B),(D)$ and $(F)$. Allocation is the percentage of radiobiosynthesized total lipid measured in Fig 1 that was found in the soma or ovaries.

Figure 3. Response surfaces of enzymatic specific activities associated with lipogenesis. Data (mean $\left(\mathrm{nmol} / \mathrm{min} / \mathrm{mg}\right.$ protein)+SEM) for fat body $\mathrm{NADP}^{+}$-isocitrate dehydrogenase $\left(\mathrm{NADP}^{+}-\mathrm{IDH}\right)[\mathrm{LW}(\mathrm{f})$ morph $=$ panel A; SW morph = panel B], and ATP-citrate lyase $(A C L)[L W(f)$ morph = panel C; SW morph = panel D].

\section{Tables}

Table 1. Summaries of ANCOVA s of response variables (Full ANCOVA tables in Appendix 3)

Table 2. Coefficients of response surfaces of ovarian mass and whole-lipid biosynthesis for LW(f) and SW morphs fed 13 diets

Table 3. --As in Table 2 but for somatic lipid allocation, ovarian lipid allocation, and proportional allocation to soma

Table 4. --As in Table 2 but for IDH and ACL specific activities 